\title{
Proposta de análise fotográfica do perfil facial em cirurgia ortognática
}

\author{
A proposal for photographic analysis of the \\ facial profile in orthognathic surgery
}

\author{
José Victor Maniglia1, Fernando Drimel Molina², \\ Caroline Gabriele Marques ${ }^{3}$
}

\begin{abstract}
Maniglia JC, Molina FD, Marques CG. Proposta de análise fotográfica do perfil facial em cirurgia ortognática. Rev Med (São Paulo). 2009 jul.-dez.;88(3/4):168-74.

RESUMO: Introdução: A análise facial, na ortodontia e na cirurgia ortognática, tem sido utilizada como um recurso diagnóstico que auxilia o profissional a direcionar o tratamento, de forma a atender uma das principais motivações do paciente - a estética facial. Objetivo: proposição de demarcação de pontos de referência na face do paciente para avaliação da fotografia do perfil facial de maneira a auxiliar o profissional na avaliação da face em ortodontia e cirurgia ortognática. Métodos: foram avaliados 25 pacientes (18 do gênero feminino e 7 do gênero feminino), com idades entre 19 e 28 anos, excluídos aqueles em tratamento ortodôntico. Usou-se uma câmera fotográfica digital DSC-S750, fixada em tripé a $1 \mathrm{~m}$ do paciente. A face do paciente foi marcada com caneta dermatográfica preta nos pontos infraorbital e tragus. Após a exposição fotográfica, a imagem foi inserida no Power Point (Microsoft), uma linha uniu os pontos infraorbital e tragus e, perpendicular a esse plano, passando pelo ponto glabela uma outra linha foi desenhada para análise do posicionamento da maxila e mandíbula com relação ao plano facial. Resultados: O método proposto auxiliou significativamente no diagnóstico, estudo do prognóstico, ensino dos parâmetros para análise facial em ortodontia e cirurgia ortognática e material auxiliar nas orientações a acompanhantes e pacientes. Conclusão: O método proposto pode auxiliar no ensino, diagnóstico e no estudo do prognóstico do paciente, bem como pode ser utilizado como material auxiliar nas orientações a acompanhantes e pacientes.
\end{abstract}

DESCRITORES: Face; Estética; Ortodontia; Fotogrametria.

\footnotetext{
1. Professor Livre-Docente da Faculdade de Medicina de São José do Rio Preto - FAMERP. Médico Otorrinolaringologista do Departamento de Otorinolaringologia da Faculdade de Medicina de São José do Rio Preto - FAMERP.

${ }^{2}$ Doutor em Ciências da Saúde pela Faculdade de Medicina de São José do Rio Preto - FAMERP. Médico Otorrinolaringologista do Departamento Otorinolaringologia da Faculdade de Medicina de São José do Rio Preto - FAMERP.

${ }^{3}$ Doutora em Ciências da Saúde Faculdade de Medicina de São José do Rio Preto - FAMERP. Ortodontista do Departamento de Otorinolaringologia da Faculdade de Medicina de São José do Rio Preto - FAMERP.
}

Endereço para correspondência: Depto. ORL - FAMERP. Av. Brigadeiro Faria Lima, 5416. Universitário - São José do Rio Preto, SP, CEP: 15090-000. e-mail: cg-marques@ hotmail.com 


\section{INTRODUÇÃO}

A avaliação da morfologia craniofacial é uma ferramenta indispensável na prática clínica e na pesquisa, e pode ser obtida por diferentes meios. Um destes meios é a fotografia, que não é cara, não expõe o paciente a uma radiação potencialmente nociva e pode proporcionar uma boa avaliação da harmonia dos relacionamentos entre estruturas craniofaciais externas, incluindo a contribuição dos tecidos moles ${ }^{1,2,3}$.

A análise facial, na ortodontia e na cirurgia ortognática, tem sido utilizada como um recurso diagnóstico que auxilia o profissional a direcionar o tratamento, de forma a atender uma das principais motivações do paciente, ou seja, a estética facial. Embora a função seja essencial para a saúde do sistema estomatognático, ter uma face esteticamente harmoniosa é condição primordial para elevar a autoestima das pessoas ${ }^{4}$.

Uma vez que as técnicas sejam padronizadas, análises fotográficas podem ser um útil complemento às análises radiográficas convencionais e cefalometria, acrescentando o fato de que, como toda ciência, a Ortodontia e a cirurgia ortognática também necessitam do auxílio de dados numéricos para exprimir o conceito de beleza ${ }^{1,5,6,7}$.

Diversos aspectos na avaliação da face do indivíduo são fundamentais no planejamento: análise de tipo facial, projeção nasal, ângulo nasolabial, protrusão e espessura dos lábios, proeminência mentoniana, projeção dos malares, comprimento da linha mento-pescoço, altura do terço inferior da face e suas relações de proporcionalidade com os demais terços faciais, simetria facial, selamento labial, tonicidade da musculatura labial e mentoniana, relação das linhas médias dos arcos dentários com o plano sagital mediano, dentre outros ${ }^{8}$.

A fotografia para análise facial de perfil deveria ser realizada com o paciente em sua posição natural da cabeça. Mesmo antes da realização de um cefalograma, o clínico deve ter o senso se uma discrepância esquelética existe e qual a natureza e o grau do problema. Embora medições quantitativas não possam ser empregadas rigorosamente, orientações estéticas podem ser utilizadas para essa avaliação. Deve-se examinar detalhadamente a harmonia facial e, principalmente, a relação da projeção anterior da maxila e mandíbula com relação ao plano facial $\left.\right|^{9,10,11}$.

Emalguns pacientes, a informação cefalométrica não condiz com a realidade do caso. Além disso, diversas definições de pontos cefalométricos incluem termos diretamente relacionados com a orientação da cabeça do paciente, cujo posicionamento pode variar durante a obtenção da radiografia, podendo estar, por exemplo, em posição natural da cabeça (PNC) ou, mais comumente, com o plano de Frankfurt paralelo ao solo ${ }^{12}$.

Quando em dúvida se devemos associar o tratamento ortodôntico à cirurgia ortognática, verificamos se há um limite aceitável para o tratamento compensatório dos arcos dentários. Se os tecidos moles fornecerem uma estética aceitável, sem que a maxila ou a mandíbula estejam muito discrepantes entre si e, principalmente, não há manifestação do paciente quanto à necessidade de cirurgia ortognática, ela não deve ser feita ${ }^{13}$.

Alterações de $\pm 10^{\circ}$ na inclinação do plano de Frankfurt podem produzir efeitos significativos sobre os valores cefalométricos. Dessa maneira, a Posição natural da cabeça (PNC) deve ser utilizada para análise cefalométrica lateral, que, em adição à avaliação clínica, permite um planejamento mais seguro e mais próximo da realidade do paciente, visto que este tipo de avaliação respeita a postura assumida pelo indivíduo em seu cotidiano ${ }^{14}$.

Sabe-se que a postura corporal global interfere na posição da cabeça, que por sua vez é diretamente responsável pela postura da mandíbula e da língua na cavidade oral. Isso é, comprovado porque há, na relação do crânio com a coluna cervical, uma intervenção entre mandíbula e osso hióide. A relação entre sistema estomatognático e postura de cabeça também pode ser estabelecida se considerarmos que as duas regiões possuem algumas conexões nervosas em comum. A posição anormal da cabeça altera as relações biomecânicas craniofaciais e craniomandibulares influindo no crescimento e na postura corporal do indivíduo, sendo importante a interceptação dos desvios funcionais nos seus estágios iniciais ${ }^{15,16,17}$.

\section{OBJETIVO}

O Objetivo desse trabalho é a proposição de demarcação de pontos de referência na face do paciente para avaliação da fotografia do perfil facial de maneira obter uma análise fotográfica do perfil facial mais próxima da análise cefalométrica para auxiliar o profissional na avaliação da face do paciente em ortodontia e cirurgia ortognática.

\section{MATERIAL E MÉTODO}

A casuística foi constituída por pacientes do Ambulatório do Serviço de Cirurgia Ortognática do Departamento de Otorrinolaringologia e Cirurgia de Cabeça e Pescoço da Faculdade de Medicina de São José do Rio Preto. 
Foram avaliados 25 pacientes, sendo excluídos aqueles que estavam em tratamento ortodôntico. Destes, 18 eram do gênero feminino e 7 do gênero masculino. A idade variou de 19 a 28 anos. A freqüência da coleta foi realizada no segundo atendimento no próprio ambulatório.

Para esta proposta de documentação foi utilizada a câmera fotográfica digital Sony, modelo DSC-S750, pela facilidade de rápida visualização, boa qualidade de imagem e fácil acesso ao profissional.

Para as fotos do perfil facial, fixamos a câmera em um tripé a uma distância de $1 \mathrm{~m}$ do paciente, que permaneceu sentado na frente do terapeuta. A centralização da fotografia foi feita no terço médio da sua face.

As marcas na face do paciente foram feitas com caneta dermatográfica preta e marcados os pontos infraorbital (Figuras 1 e 2) e tragus (Figuras 2 e 3$)$.

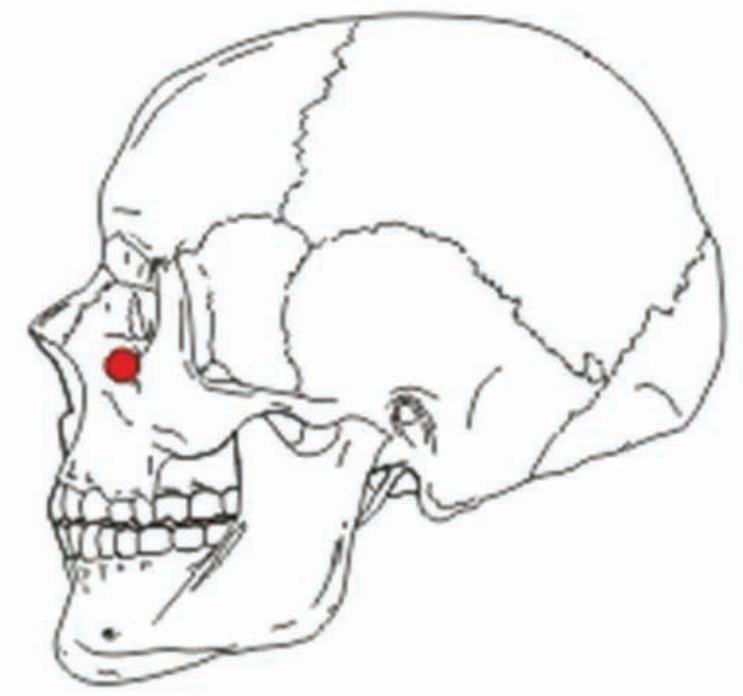

FIGURA 1. Ponto intra-orbital

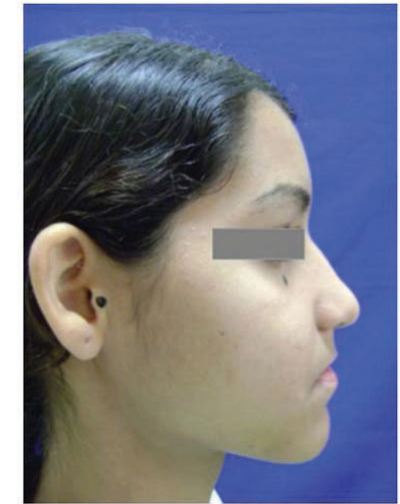

FIGURA 2. Pontos Tragus e Infraorbital demarcados na face da paciente

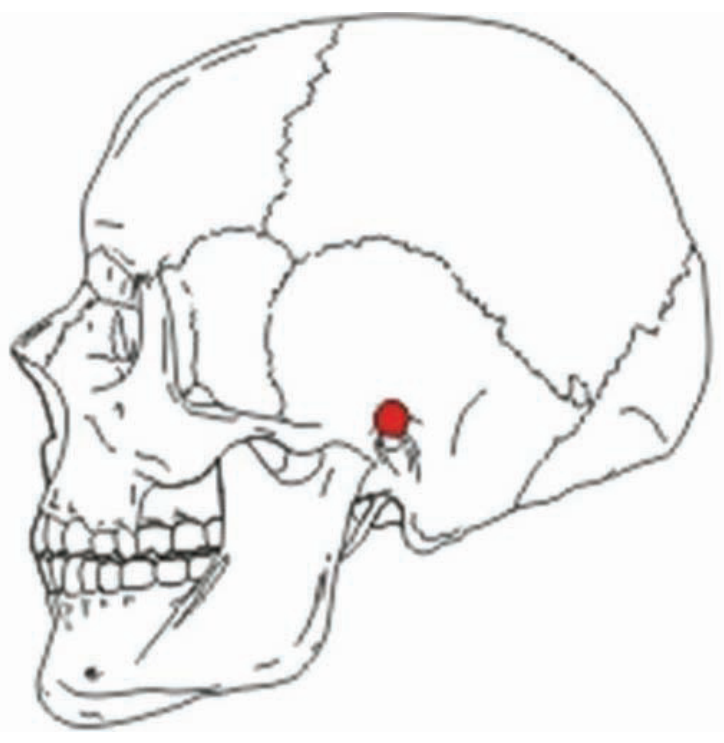

FIGURA 3. Ponto Tragus

A iluminação deficiente ou superexposição da luz podem provocar sombras que acentuam pregas $e$ cicatrizes, distorcendo uma realidade. Recomendase o flash eletrônico ou adaptar a sala como estúdio fotográfico para tomadas de fotografias pré e póstratamento.

Em relação ao fundo fotográfico, o mesmo precisa ser liso, uniforme e opaco para evitar reflexos.

No enquadramento da fotografia científica o motivo principal deve ficar no centro da moldura e nunca em posição excêntrica.

Alguns cuidados foram tomados no posicionamento do paciente para uma adequada padronização fotográfica. O mesmo manteve a cabeça posicionada com seu olhar dirigido horizontalmente ${ }^{17}$.

É importante lembrar que para as fotografias da face e pescoço, a região cervical deve estar sempre descoberta, o paciente não deve portar óculos, boné, brincos, colar, jóias, lenço ou qualquer outro objeto e as mulheres não devem usar maquiagem.

Após a exposição fotográfica, a imagem foi inserida no aplicativo Power Point (Microsoft), com o auxilio de uma linha os pontos infraorbital e tragus foram unidos e, perpendicular a esse plano, passando pelo ponto glabela (Figura 4), perpendicular à linha que uniu os pontos infra-orbital e tragus uma outra linha foi desenhada para análise do posicionamento da maxila e mandíbula com relação ao plano facial (Figuras 5 e 6 ). 


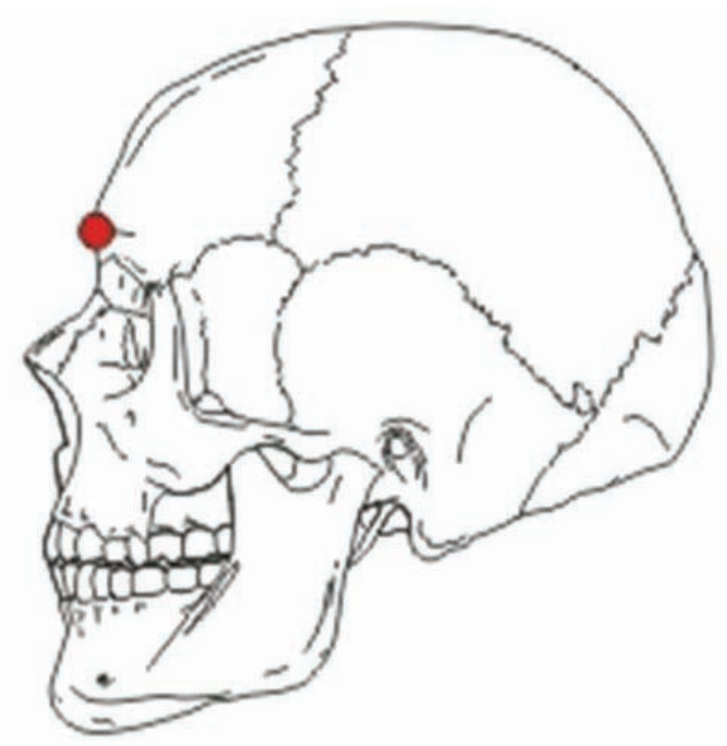

FIGURA 4. Ponto glabela

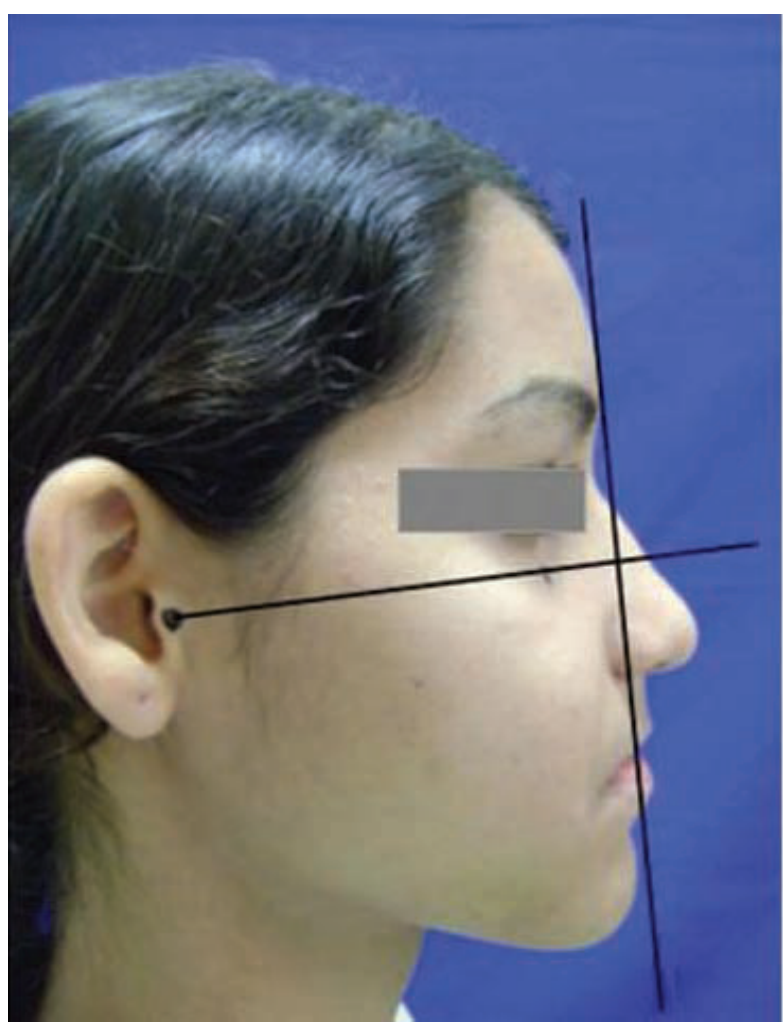

FIGURA 6. Linha unindo os pontos infraorbital e tragus e identificação do plano facial

Todas as fotografias do perfil facial dentro da tomada fotográfica proposta foram comparadas com a cefalometria (Figura 7) do paciente e com a fotografia feita com o indivíduo na posição natural da cabeça (Figura 8).

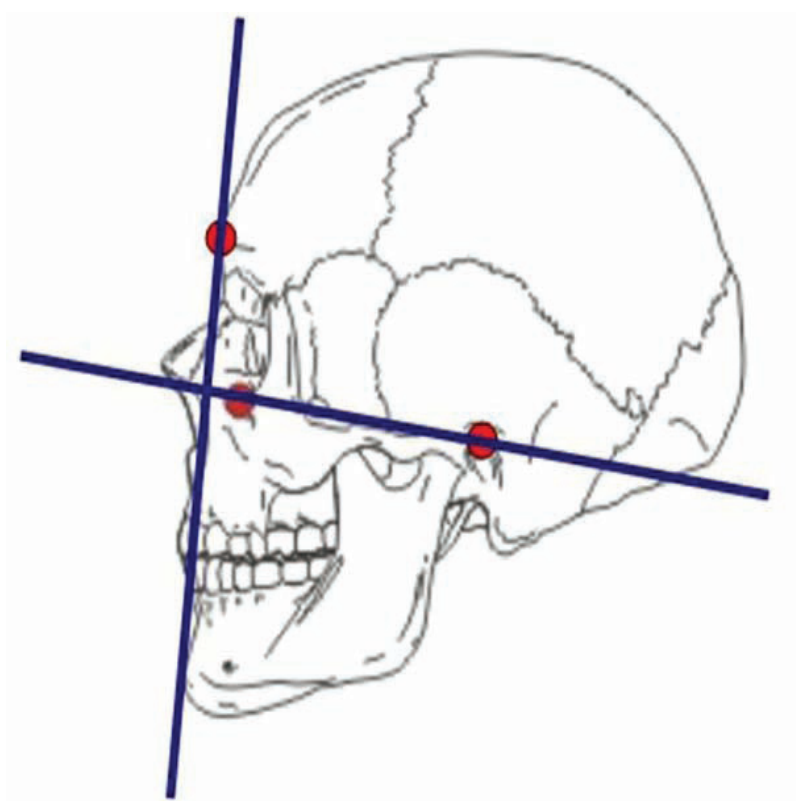

FIGURA 5. Linha unindo os pontos infraorbital e tragus e identificação do plano facial

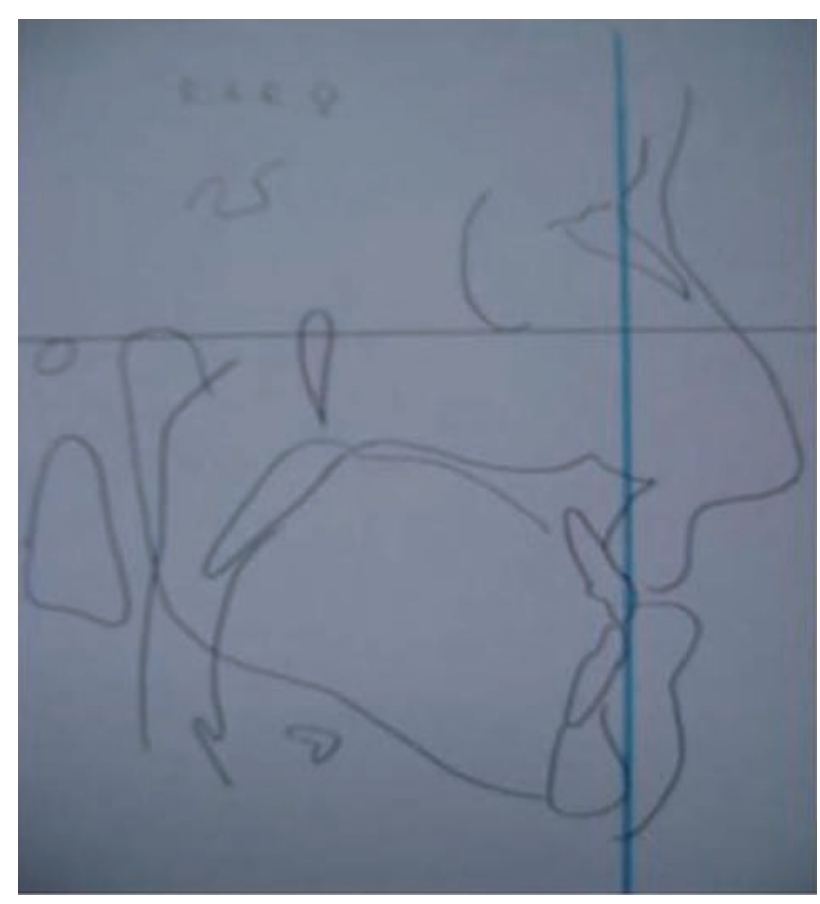

FIGURA 7. Cefalometria

\section{RESULTADOS}

$\mathrm{Na}$ análise das fotografias de perfil facial tomadas com marcações nos pontos infraorbital e tragus para posterior identificação do plano 


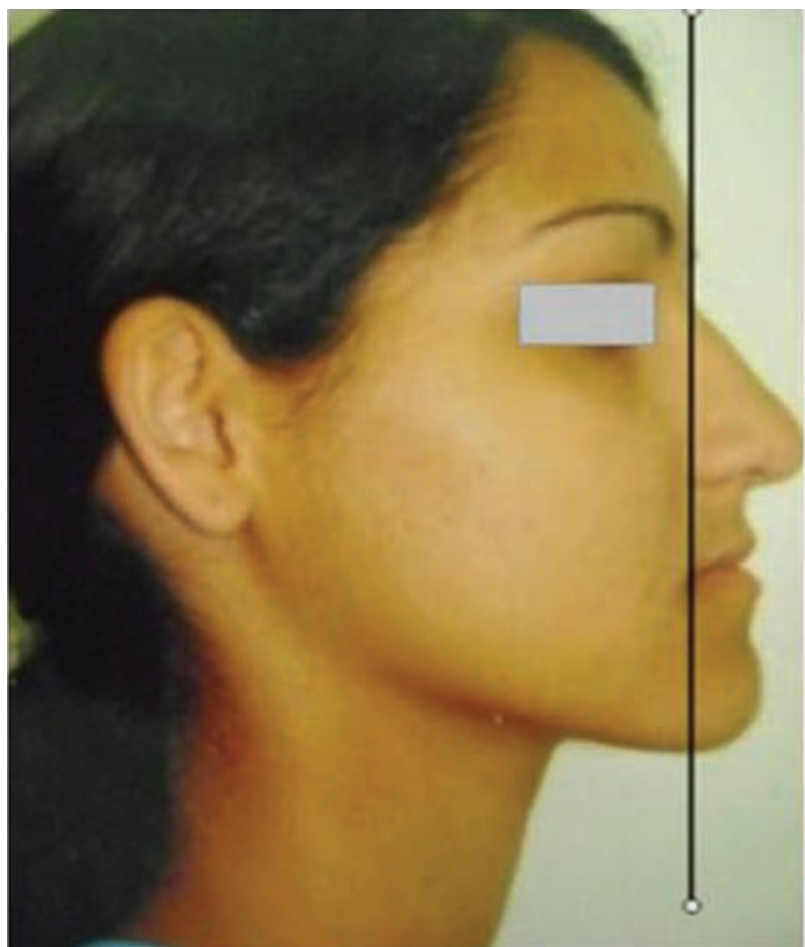

FIGURA 8. Paciente com as marcações na fotografia tomada em posição natural da cabeça sem as marcações propostas

facial e análise facial observamos os mesmos resultados encontrados na cefalometria dos pacientes analisados em referências de perfil facial (maxila retroposicionada e mandíbula bem posicionada com relação ao plano facial).

Quando comparamos a cefalometria e a fotografia comumente encontrada nas documentações ortodônticas - do perfil facial - observamos que os achados de perfil facial identificados nas fotografias sem marcadores foram diferentes da cefalometria, ou seja, maxila e mandíbula protruídas com relação ao plano facial.

\section{DISCUSSÃO}

Um dos maiores obstáculos para a quantificação das alterações de tecido mole associadas à cirurgia ortognática tem sido a inabilidade de uma análise bidimensional, como a cefalometria, em descrever precisamente estruturas tridimensionais. Devido a esse fato, várias formas de análises voltadas à cirurgia ortognática foram propostas, buscando relacionar adequadamente medidas lineares e angulares.

Os primeiros estudos produziram médias de proporções relacionando tecidos esqueléticos e tecidos moles, as quais foram empregadas na previsão de várias alterações esqueléticas e dentárias decorrentes do tratamento. No entanto, observou-se que a variabilidade individual é significativa, indicando que vários fatores podem interferir na resposta esperada, os quais não devem ser ignorados ${ }^{18}$.

Os achados desse estudo permitiram observar que há maior facilidade para o ortodontista e o cirurgião analisarem fotografias que apresentem marcas de referência de pontos craniométricos de maneira que o mesmo possa, durante a avaliação facial e fotográfica relacionar a maxila e a mandíbula com a base do crânio do paciente analisado.

Observamos também que essa demarcação facilita o aprendizado da análise facial, pois apresenta dados semelhantes aos resultados encontrados na análise cefalométrica. $O$ estudante, de posse de pontos de referência previamente identificados tem maior amplitude dos quesitos a serem identificados na análise facial, alem de se atentar para a posição da cabeça e visualização do indivíduo como um todo.

Essa modificação é de fácil aplicação e bastante simples tanto para o terapeuta como para o paciente. É feita com caneta dermatográfica e o paciente facilmente a retira após a tomada da fotografia.

Conforme demonstrou a literatura, é fundamental analisar o complexo orofacial junto à postura corporal, uma vez que o sistema estomatognático não pode separar-se de sua relação com a cabeça e o pescoço, sendo eles indissociáveis, tal como uma unidade sincrônica ${ }^{15,16,17}$.

Pudemos observar nesse trabalho que alterações na posição da cabeça interferem diretamente na compreensão da face. Pois, ao alterar a posição da mandíbula no espaço, promove certamente tracionamento da pele e marcações propostas, alterando, por conseguinte toda a análise facial.

Diversos autores já relatavam a necessidade da busca de artifícios que colaborassem com a avaliação craniofacial, no sentido de torná-la mais objetiva.

As imagens registradas facilitam uma visão mais clara da face, das bases ósseas dentárias (maxila e mandíbula) e suas relações com a base do crânio, favorecendo, juntamente com demais exames complementares, a compreensão da deformidade, bem como a necessidade de intervenção cirúrgica ou não para tratamento da mesma.

\section{CONCLUSÃO}

Concluiu-se que o método de marcação fotográfico proposto apresenta maior fidedignidade com a análise cefalométrica. Pode, portanto, auxiliar no ensino, diagnóstico e no estudo do prognóstico do paciente, bem como pode ser utilizado como material auxiliar nas orientações a acompanhantes e pacientes. 
Maniglia JC, et al. Proposta de análise fotográfica do perfil facial em cirurgia ortognática.

\section{Anexo 1. Denominadores de atratividade}

Com base no artigo de Proffit $^{9}$, Macedo ${ }^{19}$, enumeram oito denominadores de atratividade ou falta de atratividade dentofacial, em que se procuram estabelecer algumas orientações sobre o planejamento ortodôntico:

1. O tamanho do nariz e do queixo produzem um grande efeito na proeminência do lábio. Para um paciente com um nariz grande e/ou mento grande, é melhor a protrusão dos incisivos, desde que não haja um aprofundamento do sulco lábio-mentual;

2. Deficiência severa da maxila ou prognatismo de mandíbula. Criam posições labiais não atrativas e podem afetar a forma do pescoço. Raramente podem ser corrigidas apenas com a Ortodontia. Raramente o tratamento com camuflagem é satisfatório e a cirurgia deveria ser considerada;

3. A deficiência mandibular moderada é sempre esteticamente aceitável. Se colocarmos a foto desse paciente no computador e simularmos o resultado de uma possível cirurgia, o perfil reto parece drasticamente melhor para o ortodontista. Para o paciente e para os pais não há uma melhora significante no perfil;

4. Um lábio superior que se inclina para trás em relação à linha vertical verdadeira (LVV) não é estético. Os incisivos superiores não devem ser retraídos a ponto de fazer com que o lábio superior fique com uma inclinação negativa em relação à LVV;

5. A falta de sulco lábio-mentual bem definido não é estético. Está normalmente associada com a contração da musculatura para ganhar selamento labial. Deve-se a um aumento na Afai ou a protrusão dos dentes. A retrusão dos dentes melhora o perfil. Na protrusão mandibular os incisivos estão retroinclinados. Devem ser descompensados no tratamento cirúrgico;

6. Uma linha do sorriso extremamente alta mostrando uma grande quantidade de gengiva não é estética. Os pacientes só se preocupam quando isso é extremo. Mostrar quantidade moderada é perfeitamente aceitável;

7. Lábio inferior evertido não é atraente. Sempre ocorre em pacientes com trespasse horizontal excessivo, onde o lábio inferior fica sob os incisivos superiores;

8. Um perfil côncavo com lábios finos é uma característica não estética. Num paciente com lábios finos, a protrusão dos incisivos deixará os lábios mais cheios.

Maniglia JC, Molina FD, Marques CG. A proposal for photographic analysis of the facial profile in orthognathic surgery. Rev Med (São Paulo). 2009 jul.-dez.;88(3/4):168-74.

ABSTRACT. Introduction: Face analysis, in orthodontics and orthognathic surgery, have been used as a diagnosis tool to assist the professional on directing the treatment, in order to meet one of the patient's main motivations - face aesthetics. Objective: suggesting reference dotting the patient's face to assess his face profile photography aiming at helping the professional to evaluate faces in orthodontics and orthognathic surgery. Methods: Twenty five patients aged 19-28 years (18 females and 7 males) were assessed. The ones undergoing orthodontic treatment have been excluded. A digital camera DSC-S750 on a three legged support fixed $1 \mathrm{~m}$ away from the patient has been used. Infraorbital and tragus areas on the patient's face have been dotted with a black dermathographic pen. After the photographic exposition, Power Point (Microsoft) Software has been used to process the image. Infraorbital and tragus dots have been connected by a line and perpendicular to this plane, another line passing through glabella, was drawn for a maxilla and jaw analysis as to the face plane. Results: The method suggested has significantly helped diagnosis, prognostic study, learning the parameters for face analysis in orthodontics and orthognathic surgery as well as support material for instructing patient and his caretakers. Conclusion: The method suggested herein has been found to help teaching, diagnosis and patient's prognostic study, as well as to be usefull as support material for instructing patient and his caretakers.

KEY WORDS: Face; Esthetics; Orthodontics; Pothogrammetry. 


\section{REFERÊNCIAS}

1. Reche R, Colombo VL, Verona J, Moresca CA, Moro A. Análise do perfil facial em fotografias padronizadas. Rev Dental Press Ortodon Ortoped Facial. 2002;7(1):3745.

2. Verona J, Moro A, Colombo VL, Reche R, Bueno MR. Análise facial frontal masculina em repouso e durante o sorriso, métodos manual e computadorizado, em fotografias padronizadas. Parte I. J Bras Ortodon Ortop Facial. 2006;11(63):379-94.

3. Colombo VL, Moro A, Rech R, Verona J, Costa GCA. Análise facial frontal masculina em repouso e durante o sorriso, métodos manual e computadorizado, em fotografias padronizadas. Parte II - Análise do sorriso. J Bras Ortodon Ortoped Facial. 2007;12:560-75.

4. Martins LF, Sacanavini MA, Maltagliati LA. Uma nova visão da análise facial frontal numérica. In: Sakai $E$, Fiuza SC, Martins NS, Domingues-Rodrigues GC, Corrêa J, organizadores. Nova visão em ortodontia ortopedia funcional dos maxilares. São Paulo: Editora Santos; 2003. p.469-75.

5. Moro A, Freitas MR, Henriques JFC, Janson G. Estudo da recidiva em pacientes com má-oclusão de classe II, tratados pela técnica do arco de canto com extrações de pré-molares pertencentes às categorias de crescimento de Petrovic. Parte 2 - Análise de Johnston Jr. J Bras Ortodon Ortoped Facial. 2006;11(63):250-73.

6. Reis S. Pergunte a um expert - Parte I. Rev Clin Ortodon Dental Press. 2007;6(4):15-24.

7. Scavone H Jr, Trevisan H Jr, Garib DG, Ferreira FV. Facial profile evaluation in japanese-brazilian adults with normal occlusions and well-balanced faces. Am J Orthod Dentofacial Orthop. 2006;129(6):721.e1-5.

8. Chew MT, Koh CH, Sandham A, Wong HB. Subjective evaluation of the accuracy of video imaging prediction following orthognatic surgery in chinese patients. J Oral Maxillofac Surg. 2008;66(2):291-6.

9. Proffit W, White JR. R, Sarver D. Contemporary treatment of dentofacial deformity. St. Louis: Mosby; 2003.

10. Ricketts R. Cephalometric synthesis: an exercise in stating objectives planning treatment with tracings of the head roentgenogram. Am J Orthod. 1960;46(9):64773.

11. Motta ATS, Brunharo IHP, Miguel JAM, Capelli Jr J, Medeiros PJD, Almeida MAO. Simulação computadorizada do perfil facial em cirurgia ortognática: precisão cefalométrica e avaliação por ortodontistas. Rev Dent Press Ortodon Ortoped Facial. 2007;12(5):7184.

12. Haiter-Neto F, Oliveira SS, Casanova MS, Caldas MP. Telerradiografias obtidas em posição natural da cabeça alteram as grandezas cefalométricas? Rev Dent Press Ortodon Ortoped Facial. 2007;12(4):117-23.

13. Martins LF, Reis SB, Scanavini MA, Vigorito JW. Comparação entre o diagnóstico ortodôntico e a expectativa do paciente em relação ao tratamento: proposta de um questionário que facilite a comunicação entre pacientes e profissionais. J Bras Ortodon Ortoped Facial. 2003;8(43):19-28.

14. Tng TT, Chan TC, Cooke MS, Hägg U. Effect of head posture on cephalometric sagittal angular measures. Am J Orthod Dentofacial Orthop. 1993;104(4):337-41.

15. César AM, Garibaldi A, Di Nino CQM, Krakauer L. A influência da postura anteriorizada da cabeça no padrão mastigatório. Rev CEFAC. 2004;6(3):259-65.

16. Silva JH, Cunha DA. Avaliação e tratamento das alterações da deglutição. In: Marchesan IQ, organizador. Tratamento da deglutição: a atuação do fonoaudiológico em diferentes países. São José dos Campos: Pulso; 2005. p.133-48.

17. Silveira MC, Sígolo C, Quintal M, Sakano E, Tessitore, A. Proposta de documentação fotográfica em motricidade oral. Rev CEFAC. 2006;8(4):485-92.

18. Gimenez CMM, Bertoz F, Gabrielli MAC, Pereira-Filho VA, Garcia I. Magro Filho O. Avaliação cefalométrica do perfil mole de pacientes face longa submetidos à cirurgia ortognática: estudo retrospectivo. Rev Dent Press Ortodon Ortoped Facial. 2006;11(6):91-103.

19. Macedo A, Moro A, Scavone H, Martins LF. A análise facial no diagnóstico e planejamento ortodôntico. OrtodontiaSPO. 2008;41(3):234-41. 